\title{
Resonant Orbital Dynamics in LEO Region: Space Debris in Focus
}

\author{
J. C. Sampaio, ${ }^{1}$ E. Wnuk, ${ }^{2}$ R. Vilhena de Moraes, ${ }^{1}$ and S. S. Fernandes ${ }^{3}$ \\ ${ }^{1}$ Universidade Federal de São Paulo (UNIFESP), 12231-280 São José dos Campos, SP, Brazil \\ ${ }^{2}$ AMU, Astronomical Observatory, 60-286 Poznan, Poland \\ ${ }^{3}$ Instituto Tecnológico de Aeronáutica (ITA), 12228-900 São José dos Campos, SP, Brazil
}

Correspondence should be addressed to J. C. Sampaio; jarbascordeiro@gmail.com

Received 21 December 2013; Accepted 17 February 2014; Published 13 April 2014

Academic Editor: Antonio F. Bertachini A. Prado

Copyright (C) 2014 J. C. Sampaio et al. This is an open access article distributed under the Creative Commons Attribution License, which permits unrestricted use, distribution, and reproduction in any medium, provided the original work is properly cited.

\begin{abstract}
The increasing number of objects orbiting the earth justifies the great attention and interest in the observation, spacecraft protection, and collision avoidance. These studies involve different disturbances and resonances in the orbital motions of these objects distributed by the distinct altitudes. In this work, objects in resonant orbital motions are studied in low earth orbits. Using the twoline elements (TLE) of the NORAD, resonant angles and resonant periods associated with real motions are described, providing more accurate information to develop an analytical model that describes a certain resonance. The time behaviors of the semimajor axis, eccentricity, and inclination of some space debris are studied. Possible irregular motions are observed by the frequency analysis and by the presence of different resonant angles describing the orbital dynamics of these objects.
\end{abstract}

\section{Introduction}

The objects orbiting the Earth are classified, basically, in low earth orbit (LEO), medium earth orbit (MEO), and geosynchronous orbit (GEO). The LEO region has a big quantity of space debris and, consequently, most of the objects are found in low earth orbits. Considering approximately 10000 cataloged objects around the earth, one can verify the distribution of the objects as $7 \%$ of operational spacecraft, $22 \%$ of old spacecraft, $41 \%$ of miscellaneous fragments, $17 \%$ of rocket bodies, and about $13 \%$ of mission-related objects. The uncatalogued objects larger than $1 \mathrm{~cm}$ are estimated in some value between 50000 and $600000[1,2]$.

In the last years, the study about space debris mitigation in LEO region has been motivated by the increasing number of this kind of object through the years. These aspects considered in the studies englobe the observation, spacecraft protection, and collision avoidance [3, 4]. The space debris are composed of aluminum from spacecraft structures, alumina from solid rocket motor exhausts, zinc, and titanium oxides from thermal control coatings and their sizes range from several meters to a fraction of a micrometer in diameter [5].
Currently, the orbital motions of the cataloged objects can be analyzed using the 2-line element set of the North American Defense (NORAD) [6]. The TLE are composed of seven parameters and epoch. These data can be compared, for example, with the mathematical model of the propagator used to describe the motion of the artificial satellite. A similar study is done for the Brazilian satellite CBERS-1 in cooperation with China. In this case, orbital perturbations due to geopotential, atmospheric drag, solar radiation pressure, and gravitational effects of the sun and the moon are considered in the numerical integration of the orbit, and the results are compared with the TLE data [7-9].

The space between the earth and the moon has several artificial satellites and distinct objects in some resonance. Synchronous satellites in circular or elliptical orbits have been extensively studied in literature, due to the study of resonant orbits characterizing the dynamics of these satellites since the 60 's (see [10-22] and references here in). In some of these works, resonant angles associated with the exact resonance are considered in the numerical integration of the equations of motion, with the purpose to describe the resonance defined by the commensurability between the 
mean motion of the artificial satellite or space debris and the earth's rotation angular velocity. However, tesseral harmonics $J_{l m}$ produce multiple resonances in the neighborhood of the exact resonance, and some variations in the orbital motions of objects are not described.

The main goal of this work is to define resonant angles and resonant periods associated with real motions, providing more accurate information to develop an analytical model that describes a certain resonance. A model to detect resonant objects and the subset of interesting resonant objects is then presented. The time behavior of orbital elements, resonant angles, and resonant periods of specified objects is analyzed. In the studies about irregular motions, a new way to determine the frequency analysis is presented.

\section{Resonant Objects Orbiting the Earth}

In this section, the TLE data are used to verify if the objects are in resonant motions and to define the resonance in which the most of objects are found [6].

The present distribution of objects indicates the commensurability between the frequencies of the mean motion $n$ of object and the earth's rotation motion. See the histogram in Figure 1.

In Figure 1, it is verified that most of the objects is in the region $13 \leq n$ (rev/day) $\leq 15$.

To study the resonant objects using the TLE data, a criterium is established for the resonant period $P_{\text {res }}$, by the condition $P_{\text {res }}>300$ days. Note that the resonant period is related to a resonant angle which can influence the orbital motion of a particular artificial satellite. The value of $P_{\text {res }}$ helps to understand the influence of each resonant angle, and this knowledge can provide a more rigorous analytical formulation of the problem. A minimum value is established for the resonant period in order to define the main resonant angles that compose an orbital motion. The resonant angles are represented by the symbol $\phi_{\operatorname{lmpq}} . P_{\text {res }}$ is obtained by the relation

$$
P_{\text {res }}=\frac{2 \pi}{\dot{\phi}_{l m p q}},
$$

and $\dot{\phi}_{\operatorname{lmpq}}$ is calculated from [10]as follows:

$$
\begin{aligned}
\phi_{\text {lmpq }} & (M, \omega, \Omega, \Theta) \\
= & (l-2 p+q) M+(l-2 p) \omega \\
& +m\left(\Omega-\Theta-\lambda_{l m}\right)+(l-m) \frac{\pi}{2},
\end{aligned}
$$

where $a, e, I, \Omega, \omega$, and $M$ are the classical Keplerian elements: $a$ is the semimajor axis, $e$ is the eccentricity, $I$ is the inclination of the orbit plane with the equator, $\Omega$ is the longitude of the ascending node, $\omega$ is the argument of pericentre and $M$ is the mean anomaly, respectively; $\Theta$ is the Greenwich sidereal time and $\lambda_{l m}$ is the corresponding reference longitude along the equator. The term $(l-m)(\pi / 2)$ in (2) is a correction factor used in some representations of the earth gravitational potential [23-26]. This term is constant and do not appear

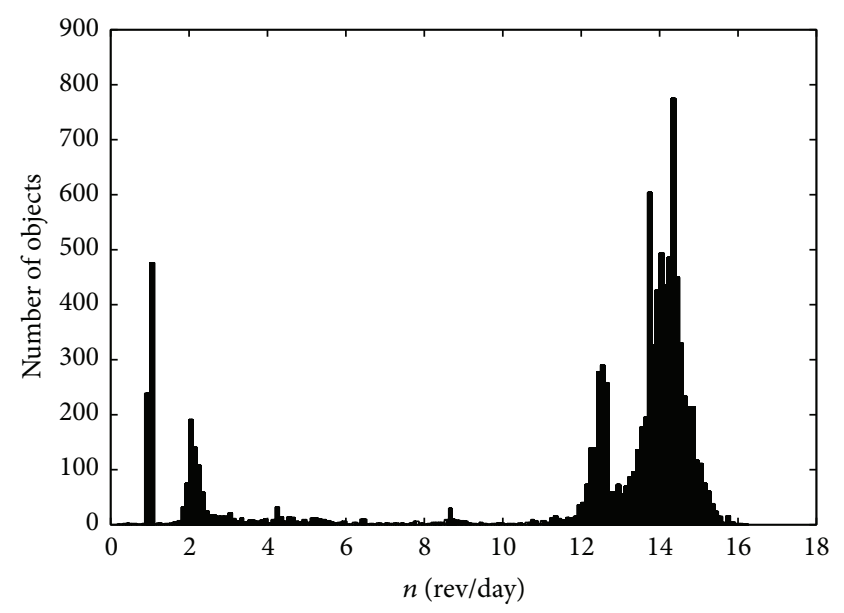

Figure 1: Histogram of the mean motion of the cataloged objects.

in $d \phi_{\text {lmpq }} / d t$ used in the calculations of the present work. So, $\dot{\phi}_{\text {lmpq }}$ is defined as

$$
\dot{\phi}_{\text {lmpq }}=(l-2 p+q) \dot{M}+(l-2 p) \dot{\omega}+m(\dot{\Omega}-\dot{\Theta}) .
$$

Substituting $k=l-2 p$ in (3), $\dot{\phi}_{k m q}$ is obtained as follows:

$$
\dot{\phi}_{k m q}=(k+q) \dot{M}+k \dot{\omega}+m(\dot{\Omega}-\dot{\Theta}) .
$$

The terms $\dot{\omega}, \dot{\Omega}$, and $\dot{M}$ can be written considering the secular effect of the zonal harmonic $J_{2}$ as $[7,27]$

$$
\begin{gathered}
\dot{\omega}=-\frac{3}{4} J_{2} n_{o}\left(\frac{a_{e}}{a_{o}}\right)^{2} \frac{\left(1-5 \cos ^{2}(I)\right)}{\left(1-e^{2}\right)^{2}}, \\
\dot{\Omega}=-\frac{3}{2} J_{2} n_{o}\left(\frac{a_{e}}{a_{o}}\right)^{2} \frac{\cos (I)}{\left(1-e^{2}\right)^{2}}, \\
\dot{M}=n_{o}-\frac{3}{4} J_{2} n_{o}\left(\frac{a_{e}}{a_{o}}\right)^{2} \frac{\left(1-3 \cos ^{2}(I)\right)}{\left(1-e^{2}\right)^{3 / 2}} .
\end{gathered}
$$

$a_{e}$ is the earth mean equatorial radius and $a_{e}=6378.140 \mathrm{~km}$, $J_{2}$ is the second zonal harmonic, $J_{2}=1.0826 \times 10^{-3}$.

The term $\dot{\Theta}$, in $\mathrm{rad} / \mathrm{day}$, is

$$
\dot{\Theta} \approx 1.00273790926 \times 2 \pi \text {. }
$$

In order to use orbital elements compatible with the way in which two-line elements were generated, some corrections are done in the mean motion of the TLE data. Considering $n_{1}$ as the mean motion of the 2-line, the semimajor axis $a_{1}$ is calculated [7]

$$
a_{1}=\left(\frac{\sqrt{\mu}}{n_{1}}\right)^{2 / 3}
$$

where $\mu$ is the earth gravitational parameter, $\mu=3.986009 \times$ $10^{14} \mathrm{~m}^{3} / \mathrm{s}^{2}$. Using $a_{1},(7)$, the parameter $\delta_{1}$ is calculated by (8) [7]

$$
\delta_{1}=\frac{3}{4} J_{2} \frac{a_{e}^{2}}{a_{1}^{2}} \frac{\left(3 \cos ^{2}(I)-1\right)}{\left(1-e^{2}\right)^{3 / 2}} .
$$


TABLE 1: 2-line data of objects orbiting the earth.

\begin{tabular}{lcc}
\hline File & Number of data & Number of objects \\
\hline alldata_2011_045 & 15360 & 9745 \\
\hline
\end{tabular}

Now, a new semimajor axis $a_{0}$, used in the calculations of the resonant period, is defined using $\delta_{1}$ from (8) [7]

$$
a_{o}=a_{1}\left[1-\frac{1}{3} \delta_{1}-\delta_{1}^{2}-\frac{134}{81} \delta_{1}^{3}\right],
$$

and the new mean motion $n_{o}$, used in the calculations, is found considering the semimajor axis corrected $a_{o}$

$$
n_{o}=\sqrt{\frac{\mu}{a_{o}^{3}}}
$$

Now, (1) to (10) can be used in a Fortran program to correct the observational data and to provide resonant angles and resonant periods related to orbital motions. The file analyzed is "alldata_2011_045" from the website Space Track [6], and it corresponds to February, 2011. In Table 1, the number of data and the number of objects are specified.

The simulation identified objects with resonant period greater than 300 days. Several values of the coefficients, $k$, $q$, and $m$ are considered in (2) producing different resonant angles to be analyzed by (1). See Table 2 showing details about the results of the simulation.

Note that Table 2 shows only the data satisfying the established criterium, $P_{\text {res }}>300$ days. The results show the resonant angles and resonant periods which compose the orbital motions of the resonant objects. Table 3 shows values of the coefficient $m$ and the correspondent number of objects. It is possible to verify that most of the orbital motions are related to the coefficient $m=14$ considering the value of the semimajor axis up to $15000 \mathrm{~km}$.

Comparing the number of objects satisfying the condition of $P_{\text {res }}>300$ days in the different regions, $a<15000 \mathrm{~km}$ and $a \geq 15000 \mathrm{~km}$, it is verified that about $62.37 \%$ of the resonant objects has $a<15000 \mathrm{~km}$. See this information in Table 4.

These studies allow to investigate the real influence of the resonance effect in the orbital dynamics of artificial satellites and space debris. The number of resonant objects in comparison with the total number of objects in the TLE data shows the great influence of the commensurability between the mean motion of the object and the earth's rotation angular velocity in its orbits. In this way, a more detailed study about the resonant period and the resonant angles is necessary.

In the next section, the orbital motions of some space debris in resonance are studied.

\section{Study of Objects in 14:1 Resonance}

Considering the results of the simulations shown in the second section, some cataloged space debris is studied with respect to the time behavior of the semi-major axis, eccentricity, inclination, resonant period, resonant angle, and the

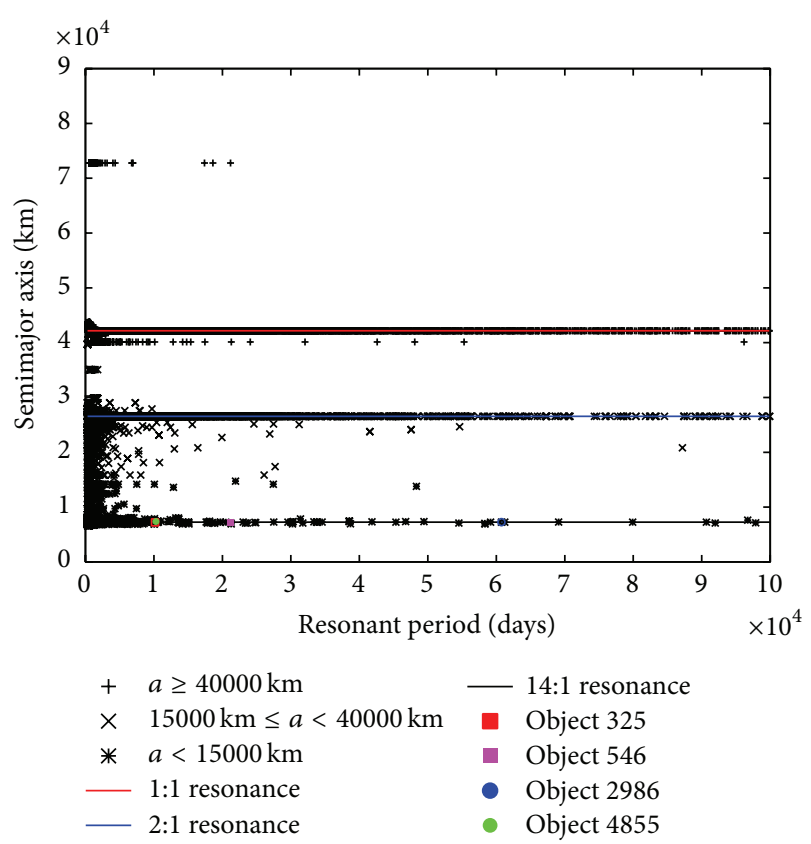

FIGURE 2: Semimajor axis versus resonant period of objects satisfying the condition $P_{\text {res }}>300$ days. The objects studied in LEO region are identified by the numbers 325, 546, 2986, and 4855 .

frequency analysis of the orbital elements. These data are analyzed in this section observing the possible regular or irregular orbital motions.

Figure 2 shows the semimajor axis versus resonant period of objects satisfying the condition $P_{\text {res }}>300$ days. The objects are distributed by the value of semimajor axis in three different regions: (1) $a<15000 \mathrm{~km}$, (2) $15000 \mathrm{~km} \leq a<$ $40000 \mathrm{~km}$, and (3) $a \geq 40000 \mathrm{~km}$.

Figure 2 shows some objects around the 1:1, 2:1 and 14:1 resonances. The orbital motions of these objects are influenced by the resonance for several years and possible irregular motions can be confined in a region delimited for resonant angles with biggest resonant periods. In order to study the orbital motions of these objects, four cataloged space debris are analyzed in the LEO region; see Figure 2. From the TLE data, objects are identified by the numbers 325 , 546,2986 , and 4855 . These objects have been chosen because they show resonant period $P_{\text {res }}>10000$ days.

Figures 3, 4, 5, and 6 show the time behavior of the semimajor axis, eccentricity, and inclination of the objects $325,546,2986$, and 4855 , and the frequency analysis of these orbital elements.

Usually, in the studies of the dynamical systems with the purpose to identify chaos, the frequency analysis is considered for a long time. But, in low earth orbits, a big number of cataloged objects have their motions influenced by different resonant periods and unknown objects can collide with each other providing more unpredictability in their motions. In this case, the frequency analysis can be more appropriate for a short time.

Several authors, [28-33], also use the frequency analysis to study the possible regular or irregular orbits in different 


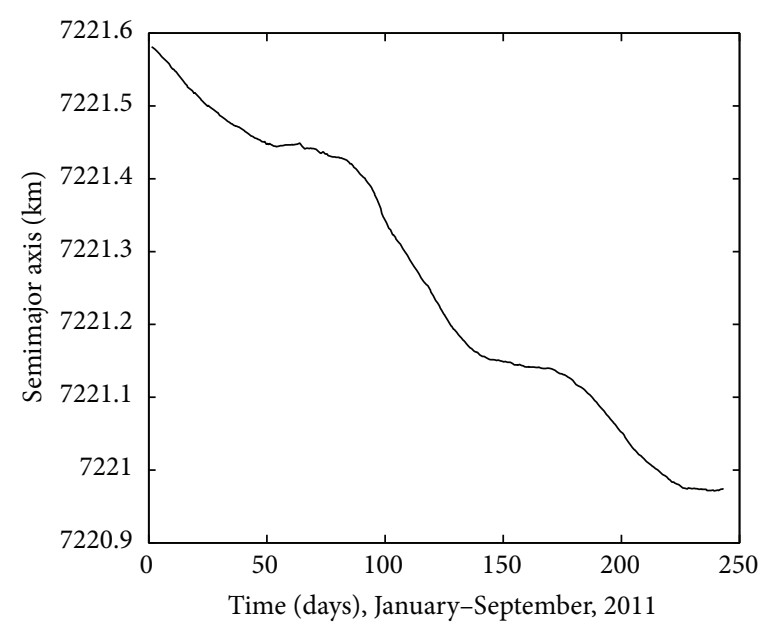

(a)

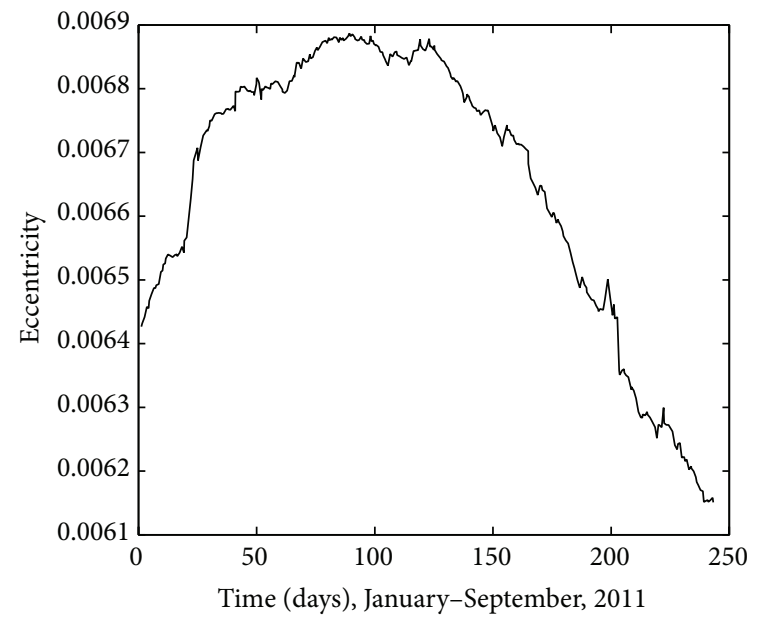

(c)

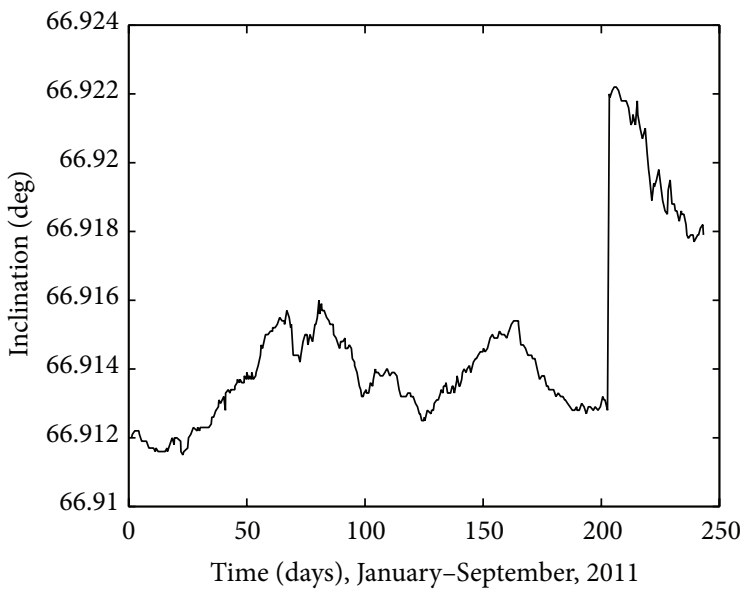

(e)

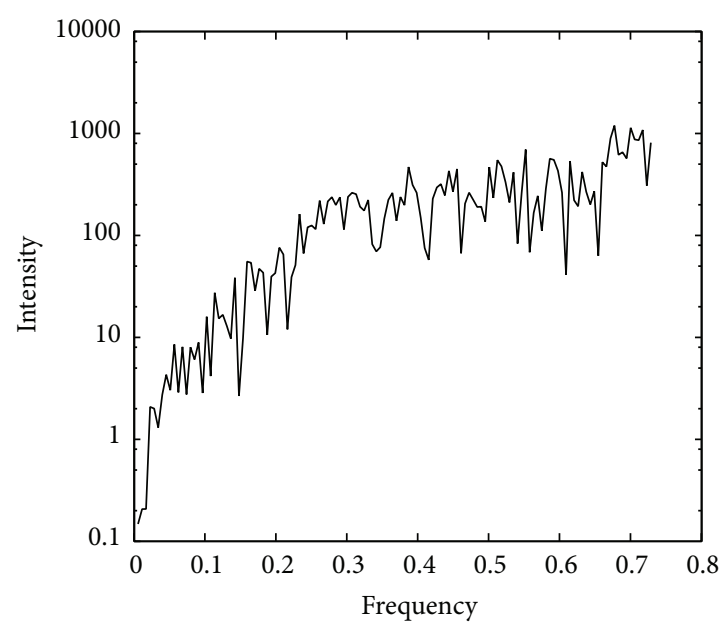

(b)

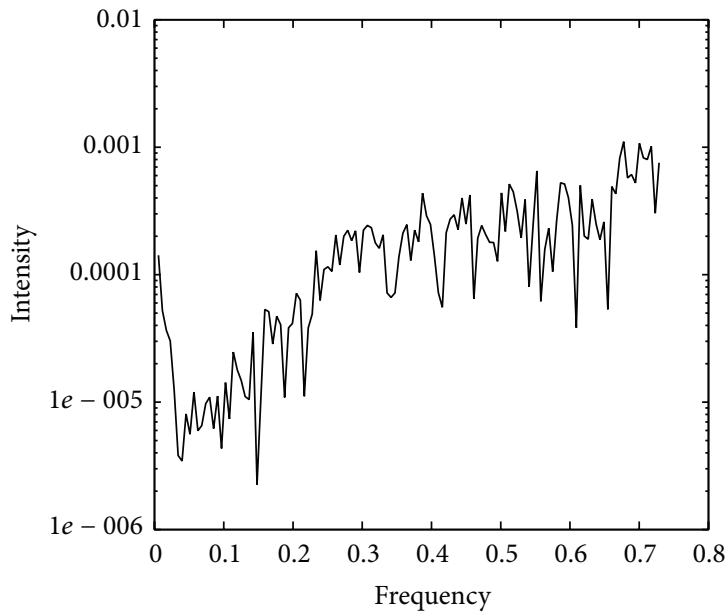

(d)

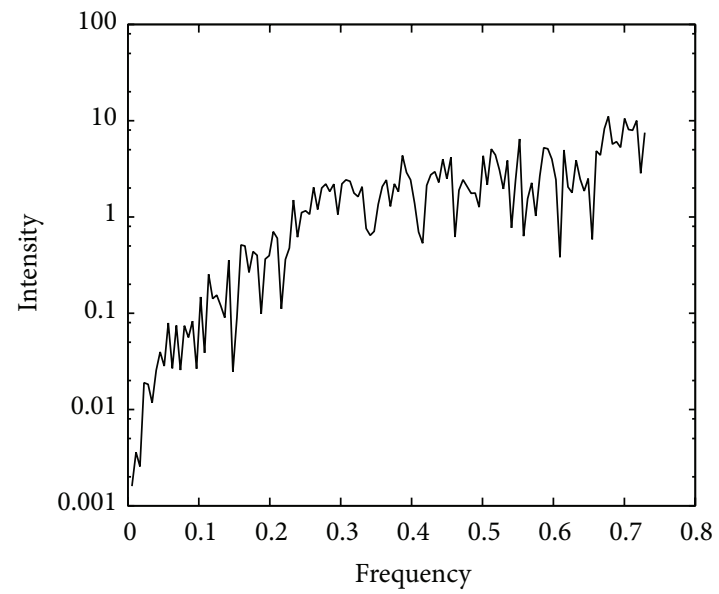

(f)

FIGURE 3: Orbital motion of object 325 corresponding to January-September, 2011: (a) time behavior of the semimajor axis, (b) power spectrum of the semimajor axis, (c) time behavior of the eccentricity, (d) power spectrum of the eccentricity, (e) time behavior of the inclination, and (f) power spectrum of the inclination. 


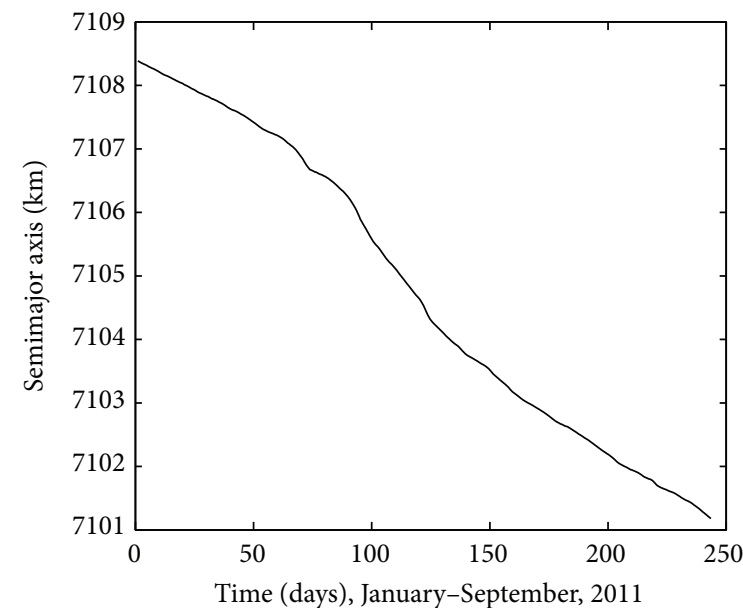

(a)

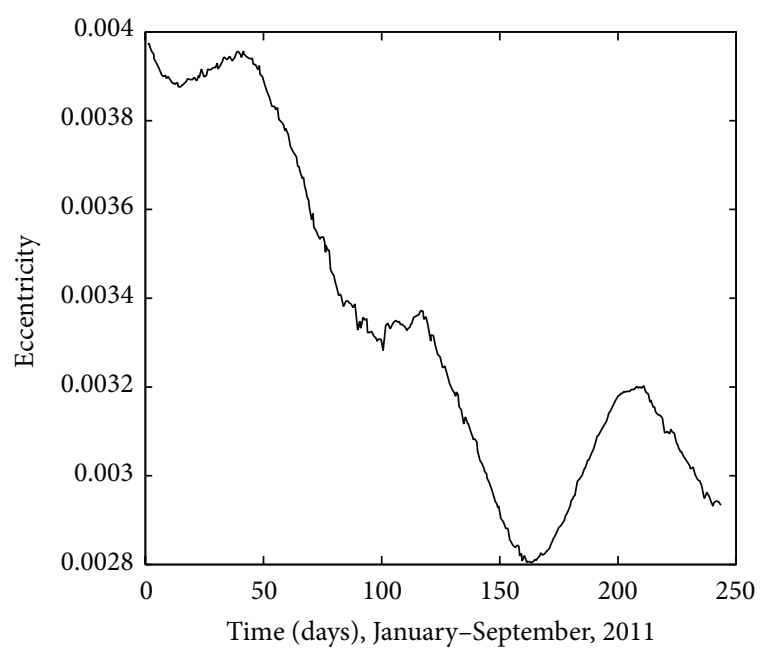

(c)

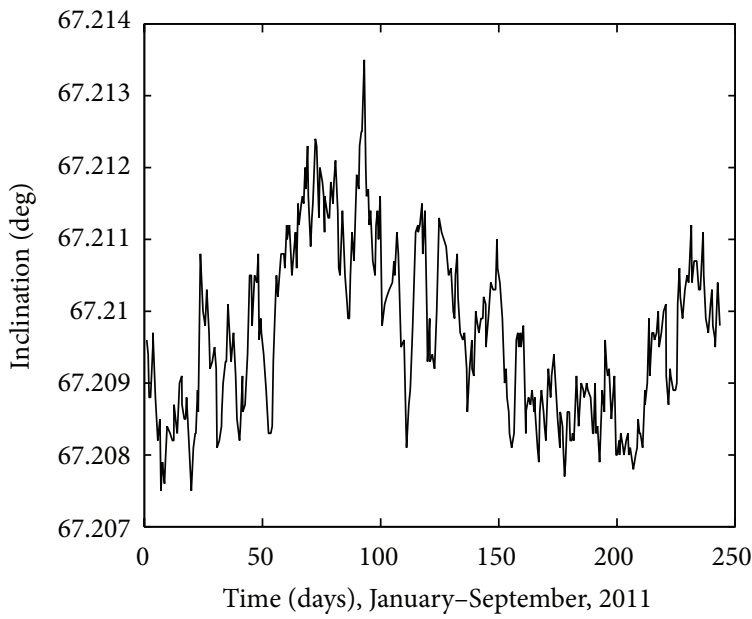

(e)

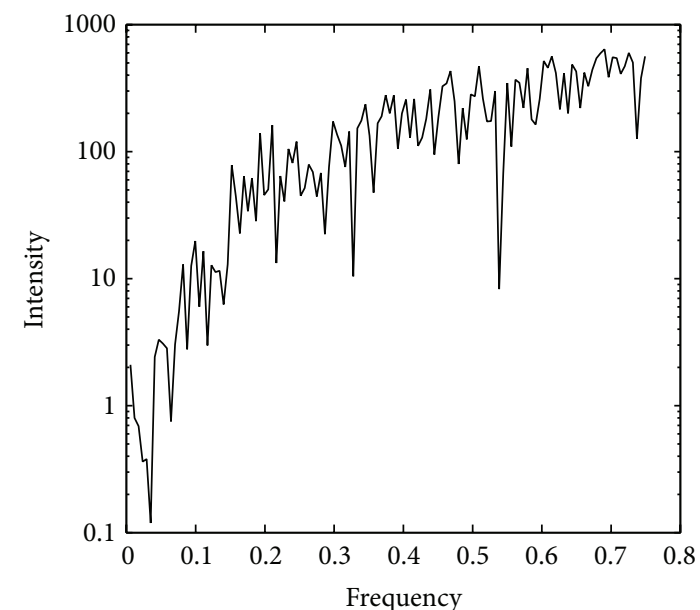

(b)

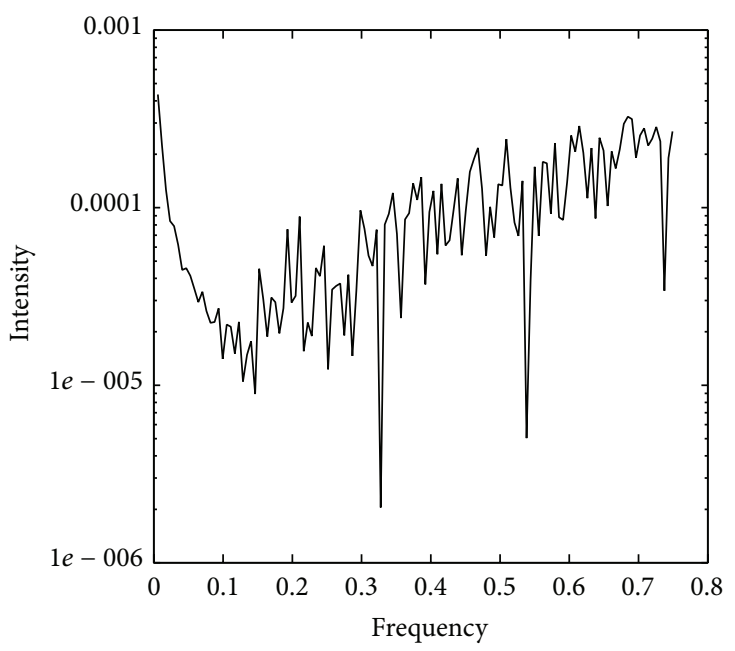

(d)

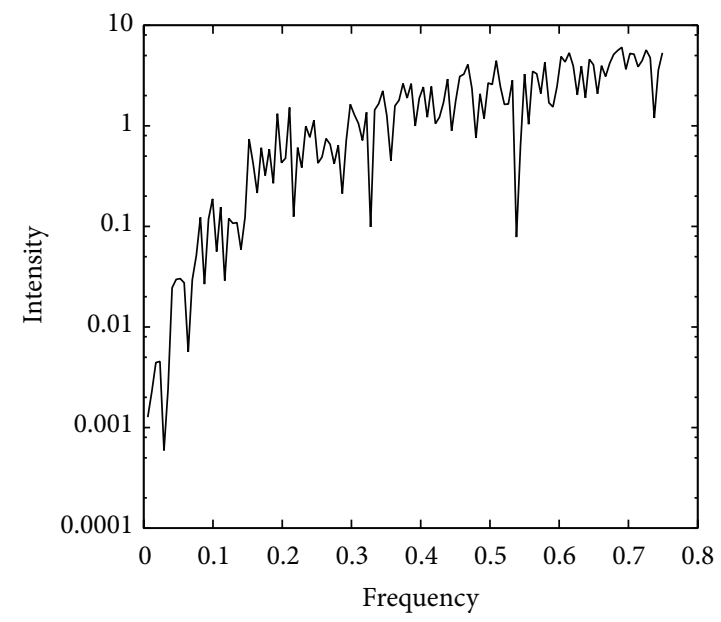

(f)

Figure 4: Orbital motion of object 546 corresponding to January-September, 2011: (a) time behavior of the semimajor axis, (b) power spectrum of the semimajor axis, (c) time behavior of the eccentricity, (d) power spectrum of the eccentricity, (e) time behavior of the inclination, and (f) power spectrum of the inclination. 


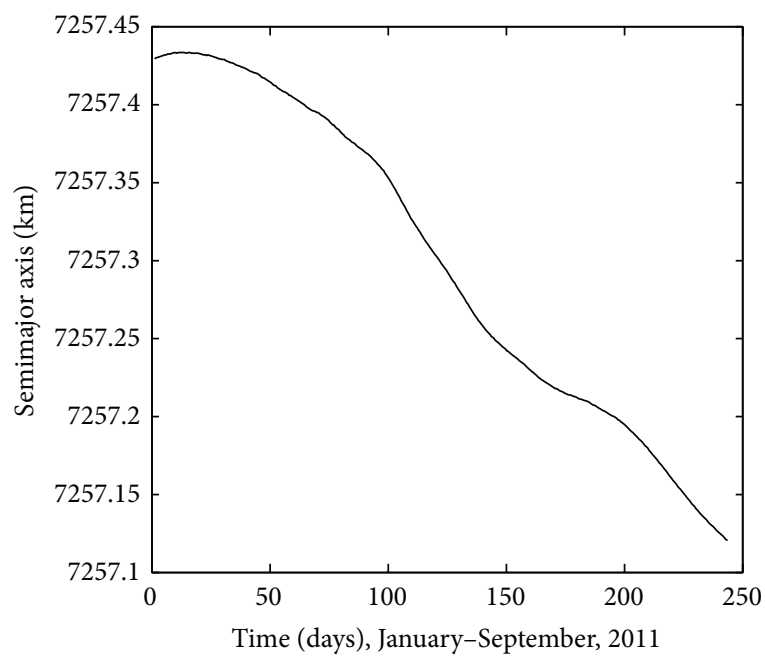

(a)

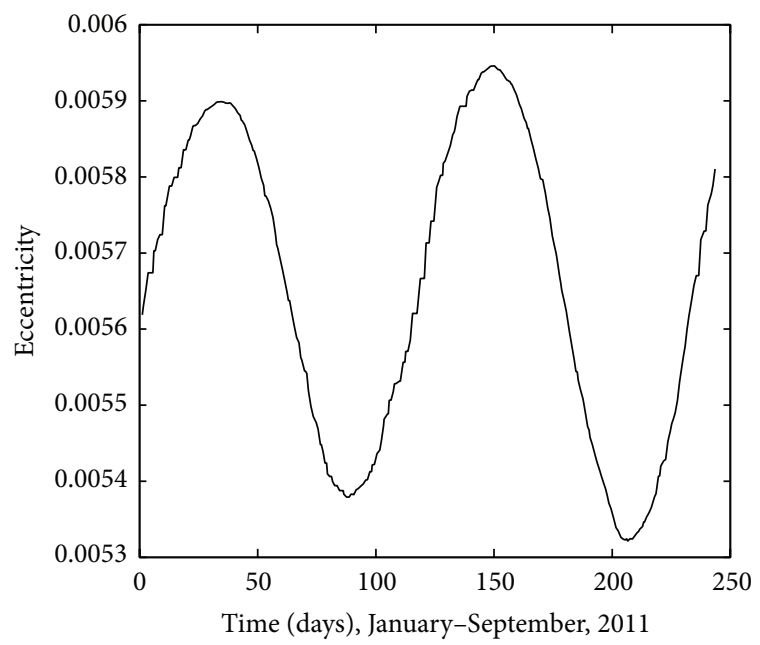

(c)

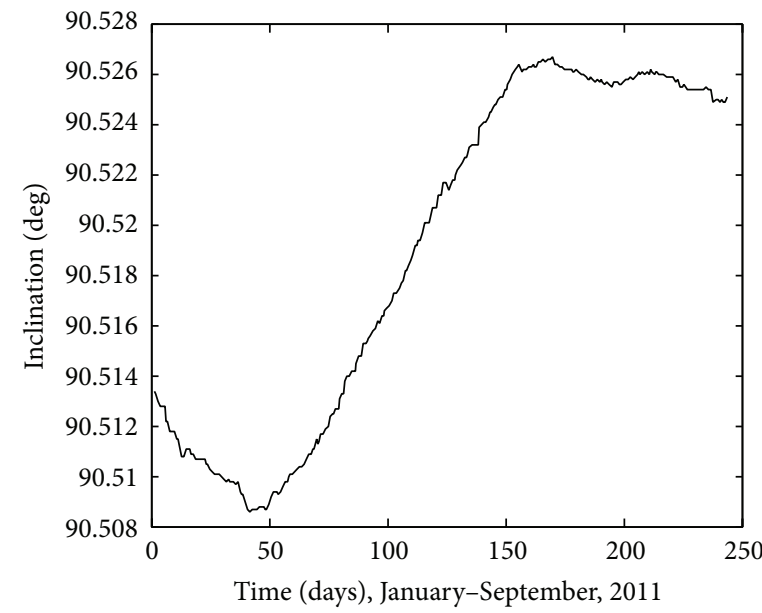

(e)

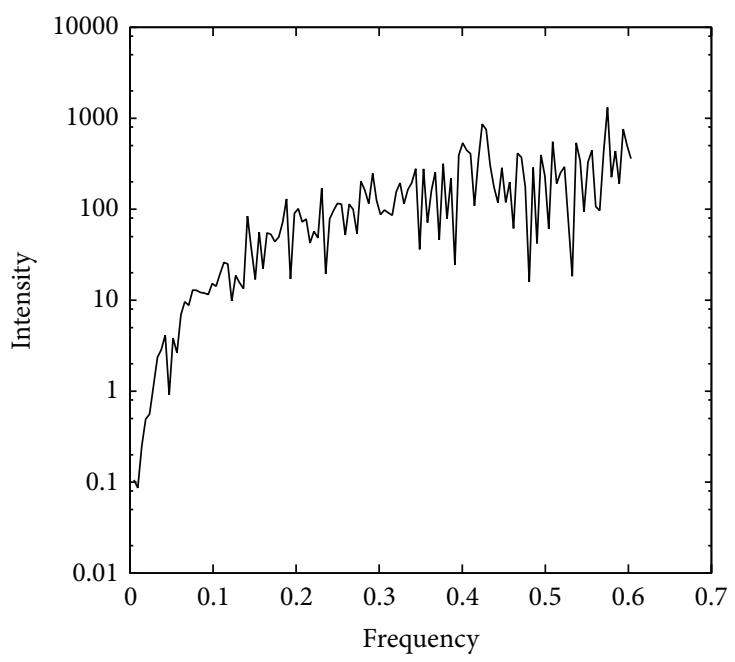

(b)

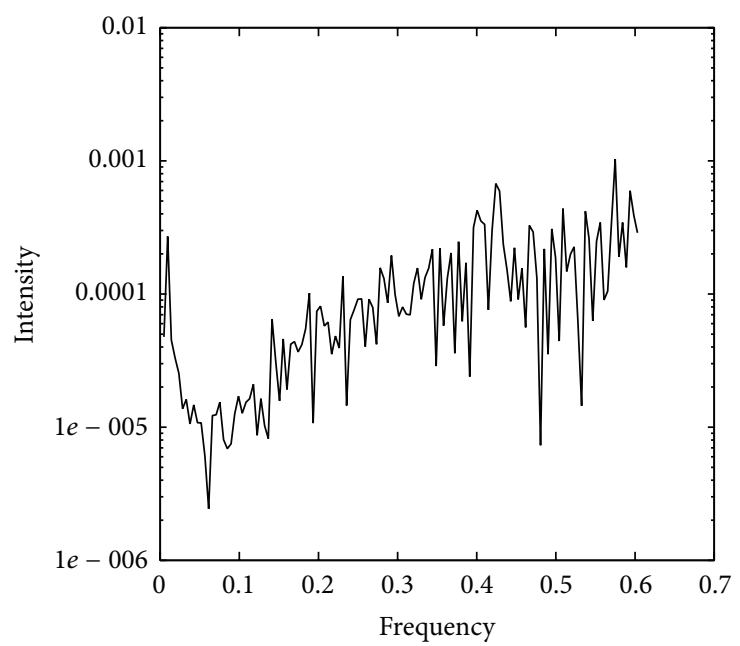

(d)

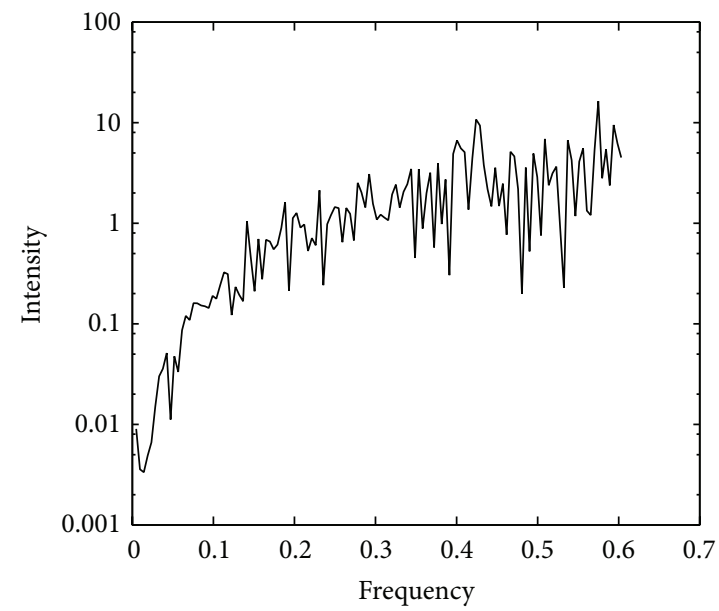

(f)

FIGURE 5: Orbital motion of object 2986 corresponding to January-September, 2011:(a) time behavior of the semimajor axis (b) power spectrum of the semimajor axis, (c) time behavior of the eccentricity, (d) power spectrum of the eccentricity, (e) time behavior of the inclination, and (f) power spectrum of the inclination. 


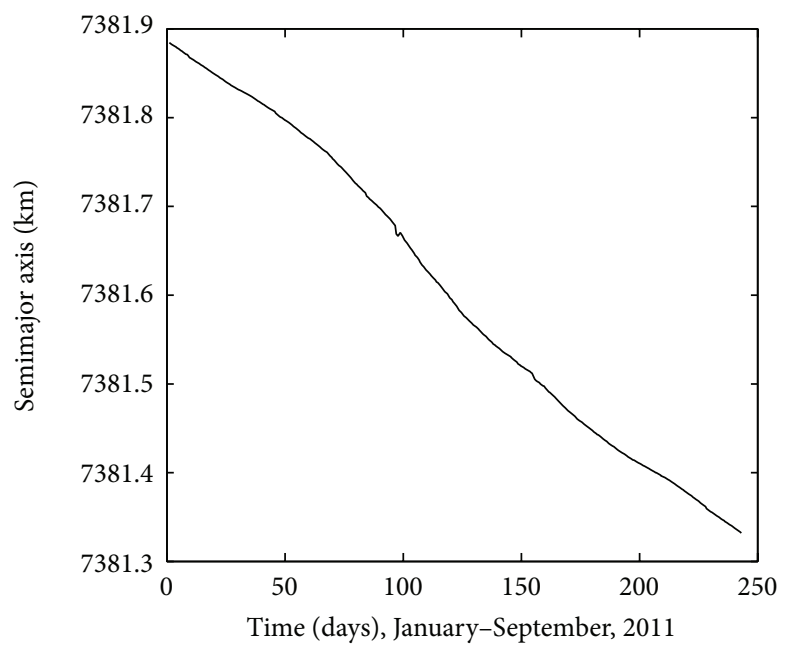

(a)

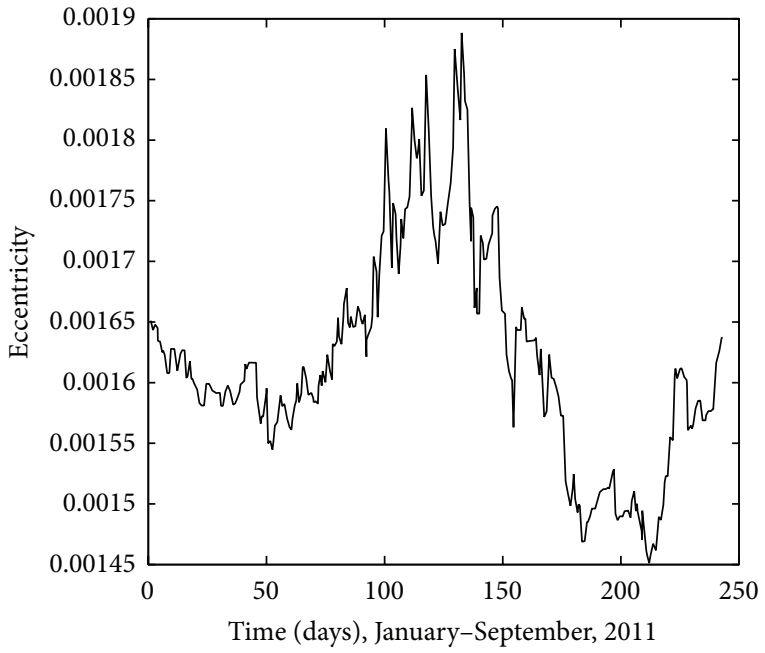

(c)

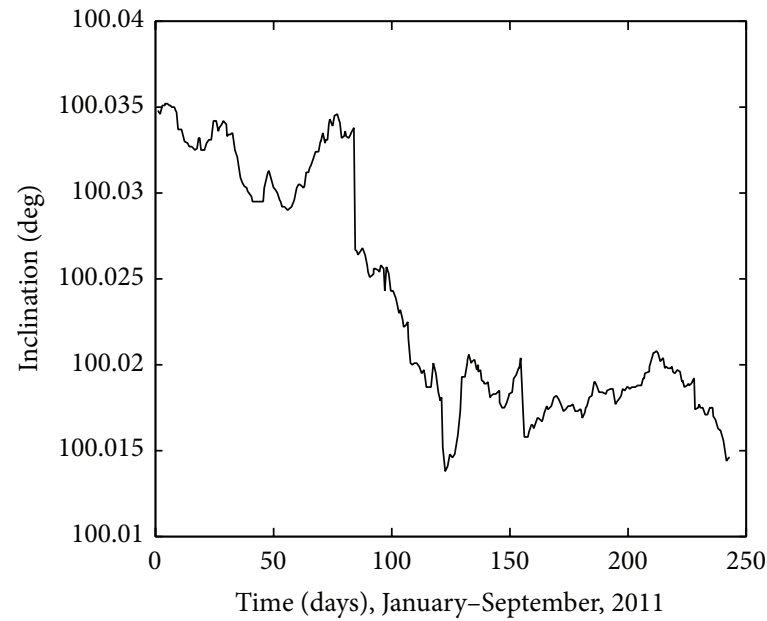

(e)

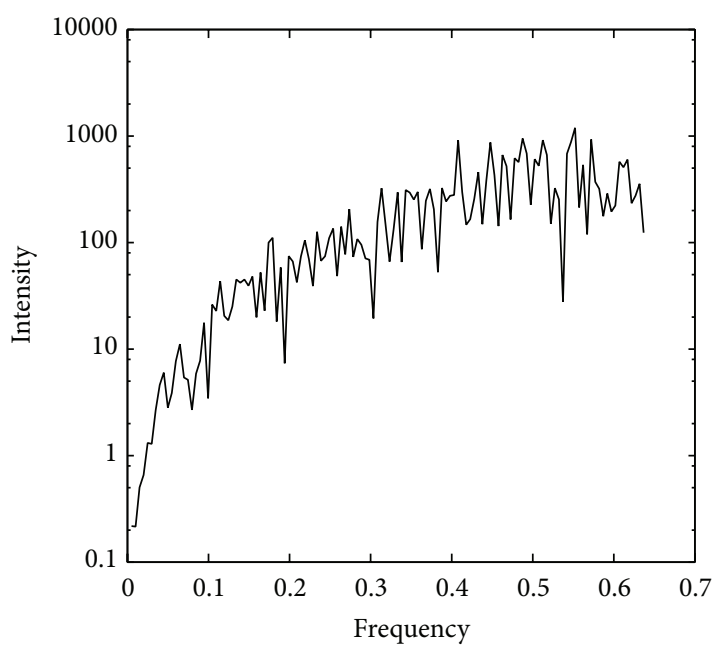

(b)

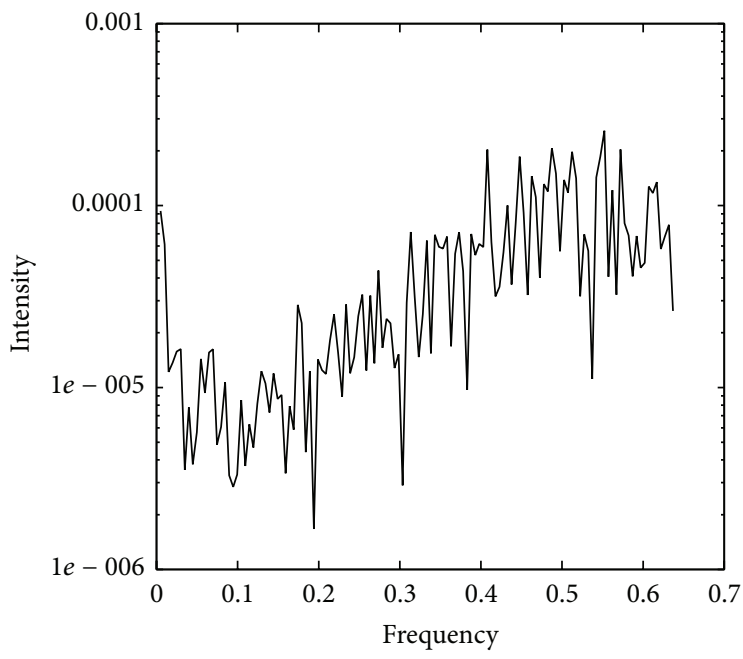

(d)

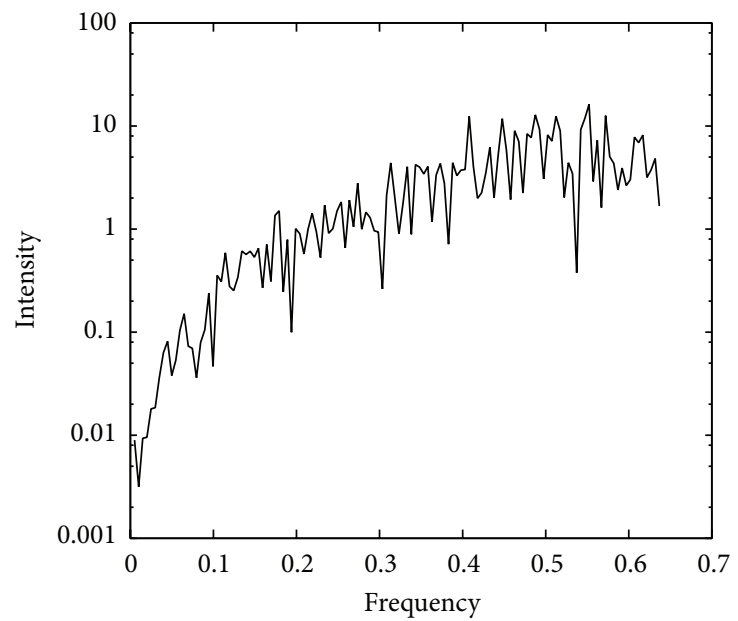

(f)

Figure 6: Orbital motion of object 4855 corresponding to January-September, 2011: (a) time behavior of the semimajor axis, (b) power spectrum of the semimajor axis, (c) time behavior of the eccentricity, (d) power spectrum of the eccentricity, (e) time behavior of the inclination, and (f) power spectrum of the inclination. 
TABLE 2: Results of the simulation: objects in resonant orbital motions.

\begin{tabular}{lcccc}
\hline Number of data & $\begin{array}{c}\text { Number of } \\
\text { different objects }\end{array}$ & Coefficient $k$ & Coefficient $q$ & Coefficient $m$ \\
\hline 3180 & 1276 & $-50 \leq k \leq 50$ & $-5 \leq q \leq 5$ & $1 \leq m \leq 50$ \\
\hline
\end{tabular}

TABLE 3: Number of objects according to the number of the coefficient $m$, considering the value of the semimajor axis up to $15000 \mathrm{~km}$.

\begin{tabular}{|c|c|}
\hline$m$ & Number of objects \\
\hline 7 & 2 \\
\hline 8 & 1 \\
\hline 9 & 3 \\
\hline 10 & 1 \\
\hline 11 & 3 \\
\hline 12 & 20 \\
\hline 13 & 31 \\
\hline 14 & 351 \\
\hline 15 & 65 \\
\hline 16 & 4 \\
\hline 18 & 3 \\
\hline 20 & 1 \\
\hline 21 & 2 \\
\hline 22 & 2 \\
\hline 23 & 2 \\
\hline 24 & 10 \\
\hline 25 & 93 \\
\hline 26 & 19 \\
\hline 27 & 66 \\
\hline 28 & 179 \\
\hline 29 & 97 \\
\hline 30 & 31 \\
\hline 31 & 13 \\
\hline 32 & 5 \\
\hline 33 & 2 \\
\hline 34 & 1 \\
\hline 35 & 4 \\
\hline 36 & 5 \\
\hline 37 & 42 \\
\hline 38 & 14 \\
\hline 39 & 14 \\
\hline 40 & 24 \\
\hline 41 & 186 \\
\hline 42 & 116 \\
\hline 43 & 114 \\
\hline 44 & 52 \\
\hline 45 & 25 \\
\hline 46 & 8 \\
\hline 47 & 4 \\
\hline 48 & 6 \\
\hline 49 & 22 \\
\hline 50 & 33 \\
\hline
\end{tabular}

dynamical systems. A brief description of the frequency analysis involving the Fast Fourier Transforms (FFT) is presented in what follows. See this method with more details in $[28,29,33]$.

Generally, the frequency analysis is applied in the output of the numerical integration in the study of dynamical systems. However, in the present work, this analysis is used
TABLE 4: Number and percentage of objects satisfying the condition $P_{\text {res }}>300$ days.

\begin{tabular}{lcc}
\hline$a<15000 \mathrm{~km}$ & $\begin{array}{c}15000 \mathrm{~km} \leq a< \\
40000 \mathrm{~km}\end{array}$ & $a \geq 40000 \mathrm{~km}$ \\
\hline 1276 & 331 & 439 \\
$62.37 \%$ & $16.18 \%$ & $21.46 \%$ \\
\hline
\end{tabular}

in the real data of the orbital motions of the objects 325,546 , 2986, and 4855, from the TLE data of the NORAD [6].

For regular motions, the orbital elements oe $(t)$ show a dependence on time as follows $[28,29,33]$ :

$$
\mathrm{oe}(t)=\sum_{h} A_{h} e^{2 \pi i \mathbf{h f} t}
$$

where $A_{h}$ represent the amplitudes, $\mathbf{h} \in \mathbf{Z}^{N}$ and $\mathbf{f}$ is a frequency vector. The components of $\mathbf{f}$ compose the fundamental frequencies of motion, and the spectral decomposition of the orbital motion is obtained from the Fourier transform when the independent frequencies are constant in the course of time [33]. It is possible to observe that $\mathbf{f}$ depends on the kind of trajectory [28].

The same numerical procedure is done for irregular and regular motions. The difference in behavior of these orbital motions is used to identify the two kinds of trajectories. The power spectrum of regular motion can be distinguished because it generally has a small number of frequency components produced by the Fourier transform. The irregular trajectories are not conditionally periodic and they do not compose an invariant tori. In this way, the Fourier transform of an orbital element, for example, is not a sum over Dirac $\delta$-functions, and consequently the power spectrum is not discrete for irregular motions [28, 33].

Observing the time behavior of the orbital elements of the objects 325, 546, 2986, and 4855 in Figures 3, 4, 5, and 6, one can verify possible regular and irregular motions in the trajectories of these space debris. The time behavior of the inclination shows irregularities. Analyzing object 325, note that, in 200 days, a fast increase in the inclination occurs, but, this variation is about $0.01^{\circ}$ and it may be related to some disturbance added to the motion.

The power spectrum of the orbital elements is not discrete and they have a big number of frequency components in Figures 3 to 6 . So, it is also important to observe if the orbital motion is influenced by different resonant angles.

Table 5 shows the resonant angles related to the orbital motions of objects $325,546,2986$, and 4855 corresponding to the period January-September, 2011. Observing Table 5, one can verify that the orbital dynamics of objects 2986 and 4855 are influenced by some resonant angles, while for the objects 


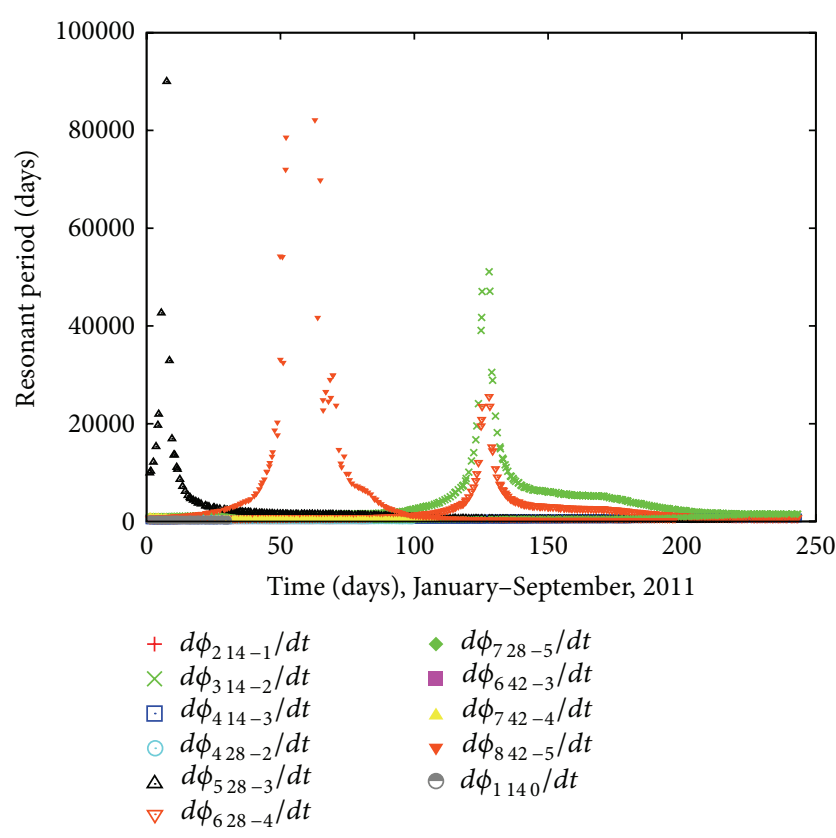

FIGURE 7: Time behavior of the resonant period corresponding to the orbital motion of object 325 .

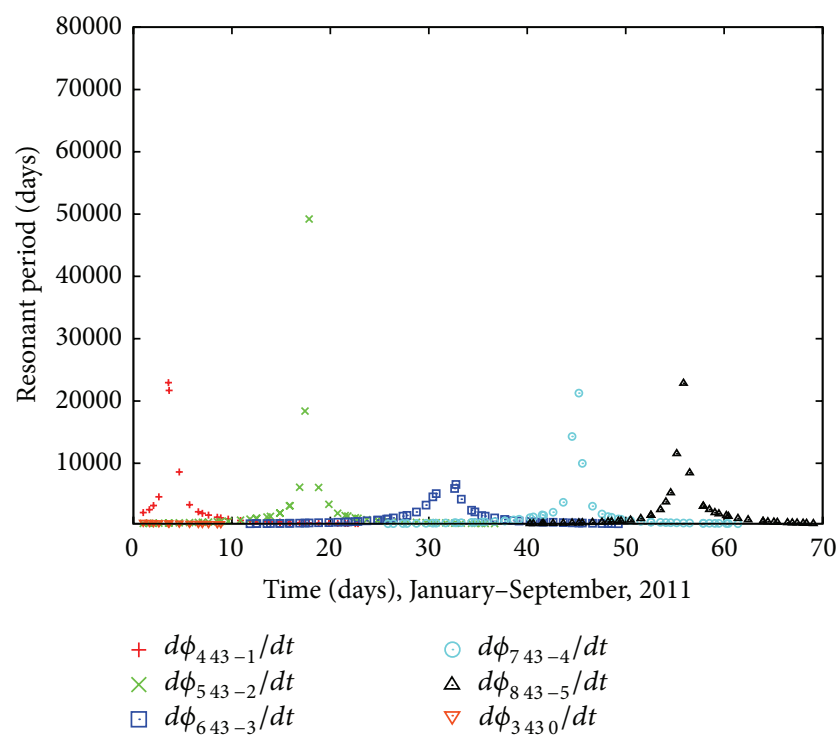

FIGURE 8: Time behavior of the resonant period corresponding to the orbital motion of object 546 .

325 and 546, several resonant angles influence their orbits simultaneously.

If the commensurability between the orbital motions of the object and the earth is defined by the parameter $\alpha$ and by the condition $\alpha=(k+q) / m$, one can say that the exact 14:1 resonance is defined by the condition $\alpha=1 / 14$. This way, by analyzing Table 5 , it is verified that the motions of objects 325 and 2986 are influenced by the exact 14:1 resonance, $\alpha=1 / 14$, while the objects 546 and 4855 are influenced by resonant angles in the neighborhood of the exact resonance.

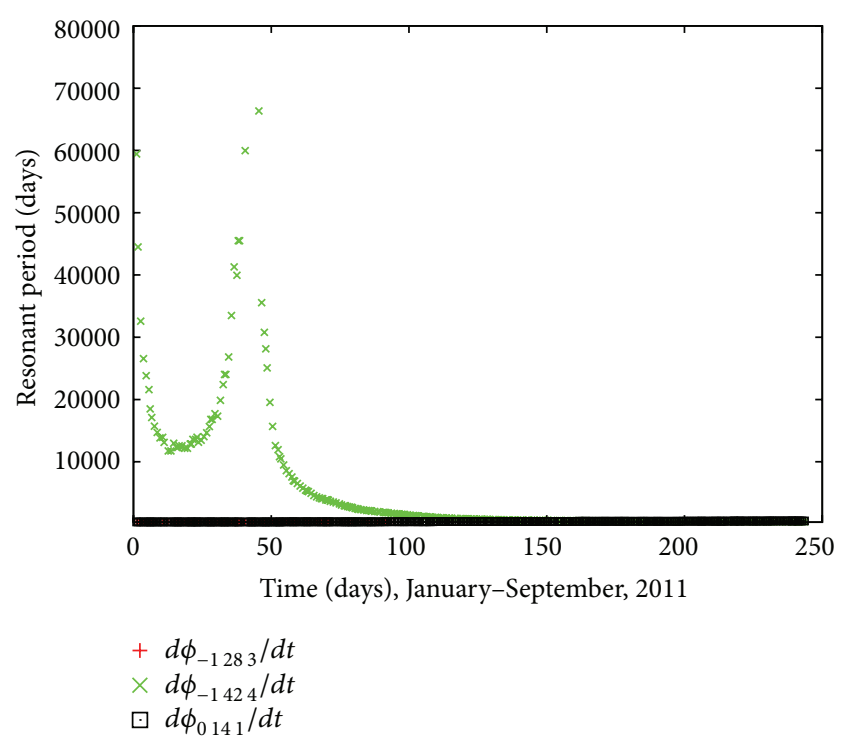

FIGURE 9: Time behavior of the resonant period corresponding to the orbital motion of object 2986.

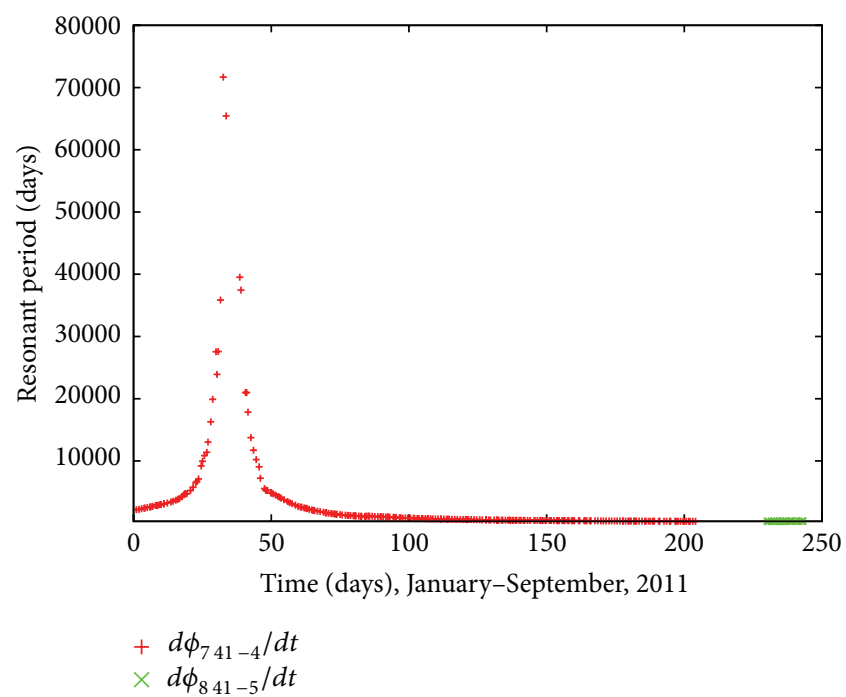

FIGURE 10: Time behavior of the resonant period corresponding to the orbital motion of object 4855 .

The condition $\alpha$ for object 546 is $\alpha=3 / 43$ and for object 4855 is $\alpha=3 / 41$.

Figures $7,8,9$, and 10 show the time behavior of the resonant period corresponding to the resonant angles presented in Table 5.

Analyzing the time behavior of the resonant period in Figures $7,8,9$, and 10 , it is verified that the resonant angles remain confined for a few days. The term confined means that the orbital motion is inside a region delimited for resonant angles with biggest resonant periods. The orbital motion of object 546 has resonant angles which satisfy the established criterium $P_{\text {res }}>300$ days for 70 days and, consequently, the orbital dynamics of this object may be losing the influence of the 14:1 resonance. Figures 9 and 10, corresponding to the 


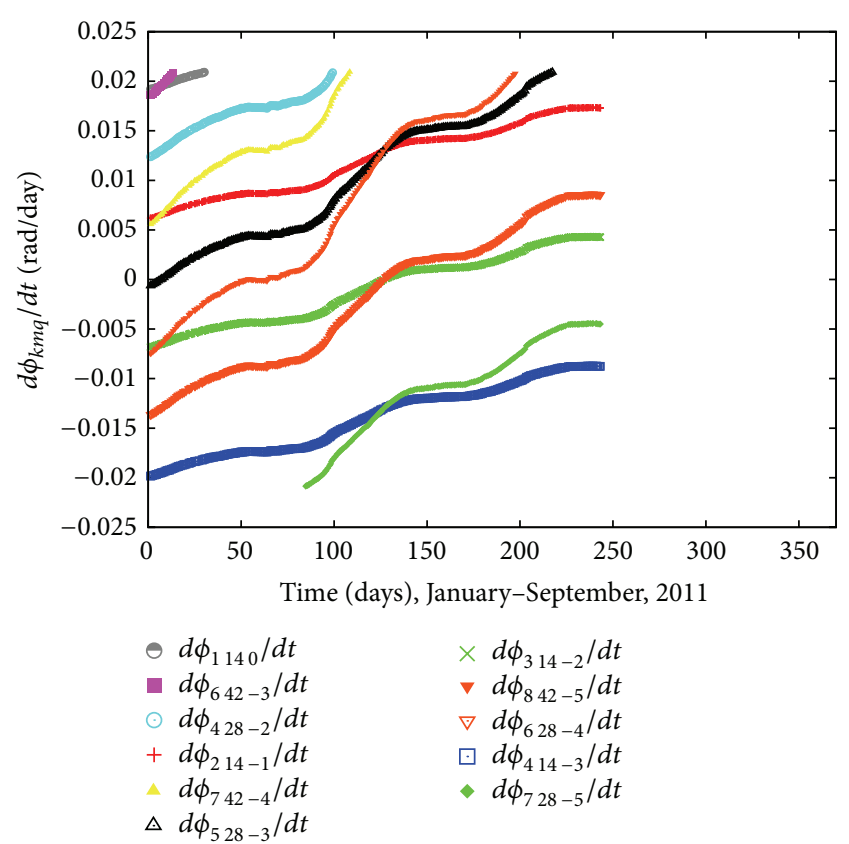

FIGURE 11: Time behavior of $\dot{\phi}_{k m q}$ corresponding to the orbital motion of object 325 .

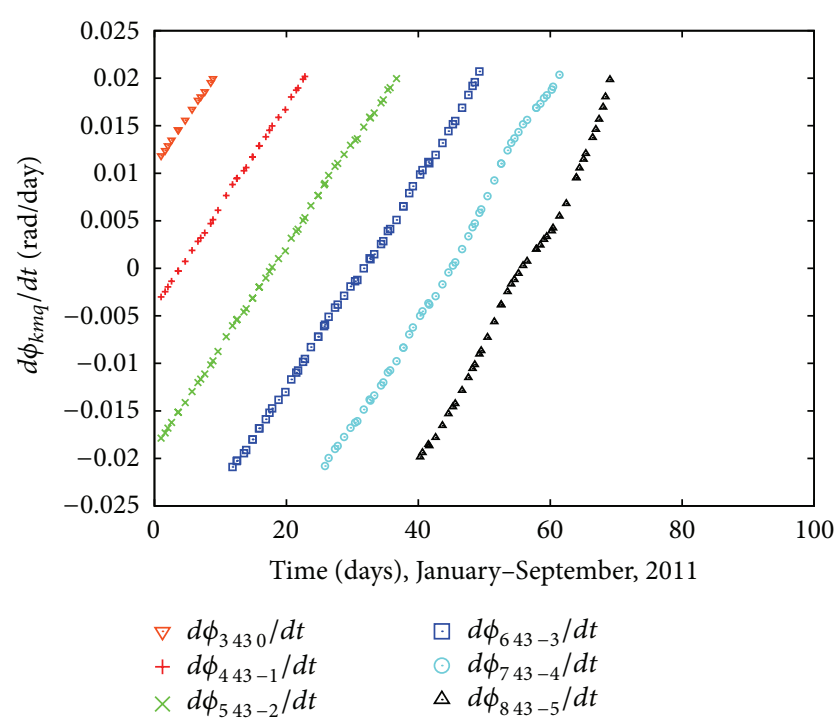

FIGURE 12: Time behavior of $\dot{\phi}_{k m q}$ corresponding to the orbital motion of object 546 .

objects 2986 and 4855, show a tendency to not remain in resonance, because the resonant period decreases from values greater than 10000 days to values smaller than 500 days. The orbital motion of object 325 is more influenced by the 14:1 resonance and this fact can be observed by the time behavior of $\dot{\phi}_{k m q}$, Figure 11.

To continue the analysis about the irregular orbital motions, the time behavior of the $\dot{\phi}_{k m q}$ is studied verifying if different resonant angles describe the orbital dynamics of these objects at the same moment. This fact can indicate

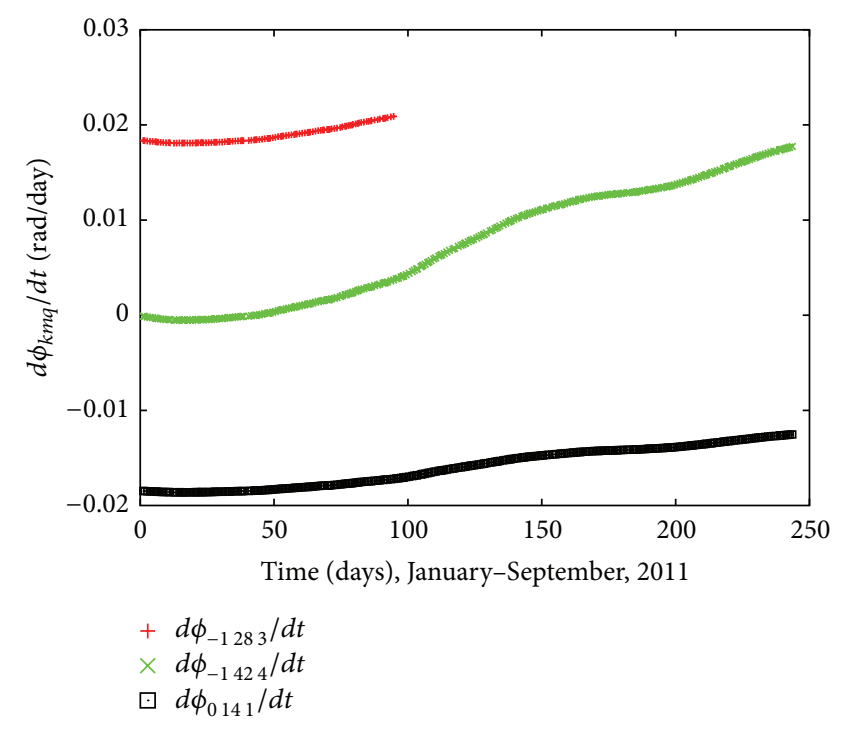

FIGURE 13: Time behavior of $\dot{\phi}_{k m q}$ corresponding to the orbital motion of object 2986 .

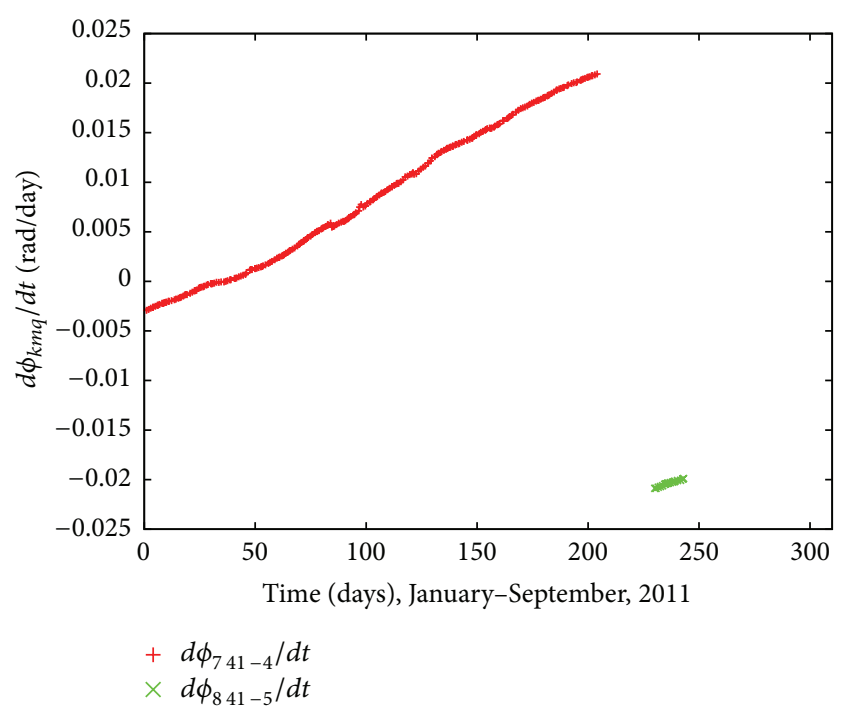

FIGURE 14: Time behavior of $\dot{\phi}_{k m q}$ corresponding to the orbital motion of object 4855 .

irregular orbits requiring a full system with all resonant angles as described in Table 5. Figures 11, 12, 13, and 14 show the time behavior of the $\dot{\phi}_{k m q}$ corresponding to the resonant angles presented in Table 5.

Analyzing the time behavior of the resonant angles $\dot{\phi}_{k m q}$ in Figures 11, 12, 13, and 14, it is possible to observe resonant angles in the exact 14:1 resonance: $\phi_{1140}, \phi_{642-3}, \phi_{428-2}$, $\phi_{214-1}, \phi_{742-4}, \phi_{528-3}, \phi_{314-2}, \phi_{842-5}, \phi_{628-4}$, and $\phi_{414-3}$ and $\phi_{728-5}$ (object 325), $\phi_{-1283}$, and $\phi_{-1424}$ (object 2986) and resonant angles in the neighborhood of the 14:1 resonance: $\phi_{3430}, \phi_{443-1}, \phi_{543-2}, \phi_{643-3}$, and $\phi_{743-4}$ and $\phi_{843-5}$ (object $546), \phi_{741-4}$, and $\phi_{841-5}$ (object 4855 ). In Figure 11, one can verify that all resonant angles have the same $\alpha$, but different values for $(k+q)$ and $m$ appear in the conditions $\alpha=(k+q) / m$, 
TABLE 5: Resonant angles $\phi_{k m q}$ related to the orbital motions of objects $325,546,2986$, and 4855 .

\begin{tabular}{lccc}
\hline Object & Coefficient $k$ & Coefficient $m$ & Coefficient $q$ \\
\hline 325 & 1 & 14 & 0 \\
325 & 6 & 42 & -3 \\
325 & 4 & 28 & -2 \\
325 & 2 & 14 & -1 \\
325 & 7 & 42 & -4 \\
325 & 5 & 28 & -3 \\
325 & 3 & 14 & -2 \\
325 & 8 & 42 & -5 \\
325 & 6 & 28 & -4 \\
325 & 4 & 14 & -3 \\
325 & 7 & 28 & -5 \\
546 & 3 & 43 & 0 \\
546 & 4 & 43 & -1 \\
546 & 5 & 43 & -2 \\
546 & 6 & 43 & -3 \\
546 & 7 & 43 & -4 \\
546 & 8 & 43 & -5 \\
2986 & -1 & 28 & 3 \\
2986 & -1 & 42 & 4 \\
2986 & 0 & 14 & -4 \\
4855 & 7 & 41 & -5 \\
4855 & 8 & 41 & \\
\hline
\end{tabular}

$\alpha=1 / 14, \alpha=2 / 28$, and $\alpha=3 / 42$. The time behavior of curves with $\alpha=2 / 28$ are similar and different of curves with $\alpha=3 / 42$ or $\alpha=1 / 14$. The orbital motion of object 546 is governed by distinct resonant angles, but, after seventy days, the influence of resonant effects decreases or disappears. Objects 325 and 2986 have their orbital motions influenced by the exact 14:1 resonance and they need a full system with different resonant angles which compose their motions. Otherwise, the orbital motion of object 4855 is defined for resonant angles separately, which can be defined as a regular orbit.

The results and discussions show the complexity in the orbital dynamics of these objects caused by the resonance effects. Furthermore, the increasing number of space debris and collisions between them can cause big problems for artificial satellites missions.

\section{Conclusions}

In this work, the orbital dynamics of synchronous space debris are studied. From the TLE data of the NORAD, objects orbiting the earth in resonant orbital motions are investigated.

Results show that some objects around the exact 1:1, $2: 1$, and 14:1 resonances remain in resonance for a long time. In other words, the orbital motions will be influenced for resonant angles for several years and possible irregular motions can be confined in a region delimited for resonant angles with biggest resonant periods.
Analyzing the cataloged objects satisfying the established criterium of the resonant period greater than 300 days, four space debris $(325,546,2986$, and 4855$)$ are studied observing the irregular characteristics in their orbits. The time behavior of the orbital elements and the respective frequency analysis are used to verify the orbits of these objects.

Several resonant angles influence, simultaneously, the orbital motions of objects 325 and 2986. The time behavior of the inclination and the frequency analysis confirm the irregularities in their orbits. The orbital dynamics of object 546 is firstly influenced by several resonant angles in the neighborhood of the 14:1 resonance, but, after some days, the regular characteristic in its orbit seems to dominate. The time behavior of the $\dot{\phi}_{k m q}$ of object 4855 shows resonant angles separately indicating a regular orbit.

The resonant orbital dynamics of artificial satellites and space debris can be described using observational data. Resonant angles which compose the orbital motions are defined before the development of analytical model, providing more accuracy. Using the same procedure shown in the present work, the resonant periods associated with resonant angles can be determined and the study about any resonance, involving the commensurability between the mean motion of object and the earth's rotation motion, can be described with more details.

Using real data, the time behavior of orbital elements allows to validate by comparison several analytical models describing perturbations in orbital motions, like atmospheric drag, solar radiation pressure, gravitational effects of the sun and the moon, for example. The frequency analysis from observational data is a new way to verify unstable regions.

In a future work, the method presented in this paper will be used to determine all resonant angles that influence a determined orbital motion and the analytical model presented in a previous paper [19]; it will be necessary to propagate the orbit, using each resonant angle and the correspondent resonant period previously determined. The description of the resonance problem is tending to a more understandable way.

\section{Conflict of Interests}

The authors declare that there is no conflict of interests regarding the publication of this paper.

\section{Acknowledgments}

This work was accomplished with support of the FAPESP under the Contracts nos. 2012/24369-0 and 2012/21023-6, SP, Brazil, CNPQ (Contract 303070/2011-0), and CAPES.

\section{References}

[1] R. Osiander and P. Ostdiek, Introduction to Space Debris, Handbook of Space Engineering, Archeology and Heritage, 2009.

[2] H. Ikeda, T. Hanada, and T. Yasaka, "Searching for lost fragments in GEO," Acta Astronautica, vol. 63, no. 11-12, pp. 13121317, 2008. 
[3] Z. Changyin, Z. Wenxiang, H. Zengyao, and W. Hongbo, "Progress in space debris research," Chinese Journal of Space Science, vol. 30, pp. 516-518, 2010.

[4] S. Nishida, S. Kawamoto, Y. Okawa, F. Terui, and S. Kitamura, "Space debris removal system using a small satellite," Acta Astronautica, vol. 65, no. 1-2, pp. 95-102, 2009.

[5] M. J. Mechishnek, "Overview of the space debris environment," Aerospace Report TR- 95(5231)-3, 1995.

[6] Space Track, "Archives of the 2-lines elements of NORAD," 2011, https://www.space-track.org/.

[7] F. R. Hoots and R. L. Roehrich, "Models for Propagation of NORAD Element Sets," Spacetrack Report 3, 1980.

[8] H. K. Kuga, "Use of the ephemeris "2-lines" from NORAD in the orbital motion of the CBERS-1 satellite," in Proceedings of the 1st Congress of Dynamics, Control and Applications, no 1, pp. 925-930, 2002.

[9] V. Orlando, R. V. F. Lopes, and H. K. Kuga, "Flight dynamics team experience throughout four years of SCD1 in-orbit operations: main issues, improvements and trends," in Proceedings of 12th International Symposium on Space Flight Dynamics, pp. 433-437, ESOC, Darmstadt, Germany, 1997.

[10] M. T. Lane, "An analytical treatment of resonance effects on satellite orbits," Celestial Mechanics, vol. 42, no. 1-4, pp. 3-38, 1987.

[11] A. Jupp, "A solution of the ideal resonance problem for the case of libration," Astronomical Journal, vol. 74, pp. 35-43, 1969.

[12] T. A. Ely and K. C. Howell, "Long-term evolution of artificial satellite orbits due to resonant tesseral harmonics," Journal of the Astronautical Sciences, vol. 44, no. 2, pp. 167-190, 1996.

[13] D. M. Sanchez, T. Yokoyama, P. I. O. Brasil, and R. R. Cordeiro, "Some initial conditions for disposed satellites of the systems GPS and Galileo constellations," Mathematical Problems in Engineering, vol. 2009, Article ID 510759, 22 pages, 2009.

[14] R. V. De Moraes and L. D. D. Ferreira, "GPS satellites orbits: resonance," Mathematical Problems in Engineering, vol. 2009, Article ID 347835, 12 pages, 2009.

[15] J. C. Sampaio, R. Vilhena de Moraes, and S. S. Fernandes, "Terrestrial artificial satellites dynamics: resonant effects," in Proceedings of the 21st International Congress of Mechanical Engineering, Natal, Brazil, 2011.

[16] J. C. Sampaio, R. Vilhena De Moraes, and S. Da Silva Fernandes, "The orbital dynamics of synchronous satellites: irregular motions in the 2:1 resonance," Mathematical Problems in Engineering, vol. 2012, Article ID 405870, 22 pages, 2012.

[17] J. C. Sampaio, R. Vilhena de Moraes, and S. S. Fernandes, "Artificial satellites dynamics: resonant effects," Journal of Aerospace Engineering, Sciences and Applications, vol. 4, no. 2, pp. 1-14, 2012.

[18] J. C. Sampaio, E. Wnuk, R. Vilhena de Moraes, and S. S. Fernandes, "The orbital motion in the LEO region: objects in deep resonance," in Proceedings of the 39th COSPAR Scientific Assembly, Mysore, India, 2012.

[19] J. C. Sampaio, A. G. S. Neto, S. S. Fernandes, R. Vilhena de Moraes, and M. O. Terra, "Artificial satellites orbits in 2:1 resonance: GPS constellation," Acta Astronautica, vol. 81, no. 2, pp. 623-634, 2012.

[20] R. Vilhena de Moraes, J. C. Sampaio, S. da Silva Fernandes, and J. K. Formiga, "A semi-analytical approach to study resonances effects on the orbital motion of artificial satellites," in Proceedings of 23rd AAs- AIAA Space Flight Meeting, Kauai, Hawaii, USA, 2013.
[21] F. Deleflie, A. Rossi, C. Portmann, G. Métris, and F. Barlier, "Semi-analytical investigations of the long term evolution of the eccentricity of Galileo and GPS-like orbits," Advances in Space Research, vol. 47, no. 5, pp. 811-821, 2011.

[22] L. Anselmo and C. Pardini, "Orbital evolution of the first upper stages used for the new European and Chinese navigation satellite systems," Acta Astronautica, vol. 68, no. 11-12, pp. 20662079, 2011.

[23] J. P. Osorio, Disturbances of satellite orbits in the study of earth gravitational field, Portuguese Press, Porto, Portugal, 1973.

[24] P. R. Grosso, Orbital motion of an artificial satellite in 2:1 resonance [M.S. thesis], ITA: Instituto Tecnológico de Aeronáutica, São Paulo, Brazil, 1989.

[25] P. H. C. N. Lima Jr., Resonant systems at high Eccentricities in the artificial satellites motion [Ph.D. thesis], ITA: Instituto Tecnológico de Aeronáutica, São Paulo, Brazil, 1998.

[26] J. K. S. Formiga and R. Vilhena de Moraes, "Dynamical systems: an integrable kernel for resonance effects," Journal of Computational Interdisciplinary Sciences, vol. 1, no. 2, pp. 89-94, 2009.

[27] J. Golebiwska, E. Wnuk, and I. Wytrzyszczak, "Space debris observation and evolution predictions, Software Algorithms Document (SAD)," ESA PECS Project 98088, Astronomical Observatory of the Adam Mickiewicz University, Poznan, Poland, 2010.

[28] G. E. Powell and I. C. Percival, "A spectral entropy method for distinguishing regular and irregular motion of Hamiltonian systems," Journal of Physics A: General Physics, vol. 12, no. 11, pp. 2053-2071, 1979.

[29] J. Laskar, "Frequency analysis for multi-dimensional systems. Global dynamics and diffusion," Physica D: Nonlinear Phenomena, vol. 67, no. 1-3, pp. 257-281, 1993.

[30] M. Sidlichovsky and D. Nesvorny, "Frequency modified fourier transform and its application to asteroids," Celestial Mechanics and Dynamical Astronomy, vol. 65, no. 1-2, pp. 137-148, 1996.

[31] N. Callegari Jr., T. A. Michtchenko, and S. Ferraz-Mello, "Dynamics of two planets in the 2/1 mean-motion resonance," Celestial Mechanics and Dynamical Astronomy, vol. 89, no. 3, pp. 201-234, 2004.

[32] N. Callegari Jr., S. Ferraz-Mello, and T. A. Michtchenko, "Dynamics of two planets in the $3 / 2$ mean-motion resonance: application to the planetary system of the pulsar PSR B1257+12," Celestial Mechanics and Dynamical Astronomy, vol. 94, no. 4, pp. 381-397, 2006.

[33] S. Ferraz-Mello, T. A. Michtchenko, C. Beaugé, and N. Callegari Jr., "Extrasolar planetary systems," in Chaos and Stability in Exrasolar Planetary Systems, R. Dvorak and J. Henrard, Eds., vol. 683 of Lectures Notes in Physics, pp. 219-271, 2005. 


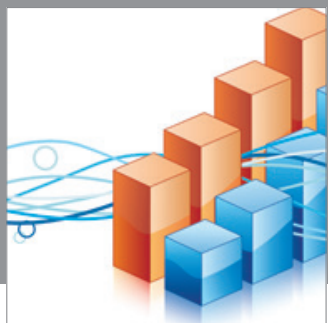

Advances in

Operations Research

mansans

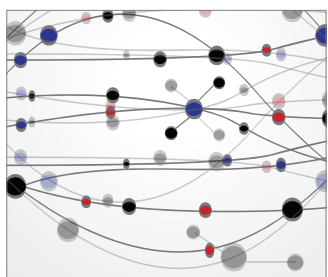

The Scientific World Journal
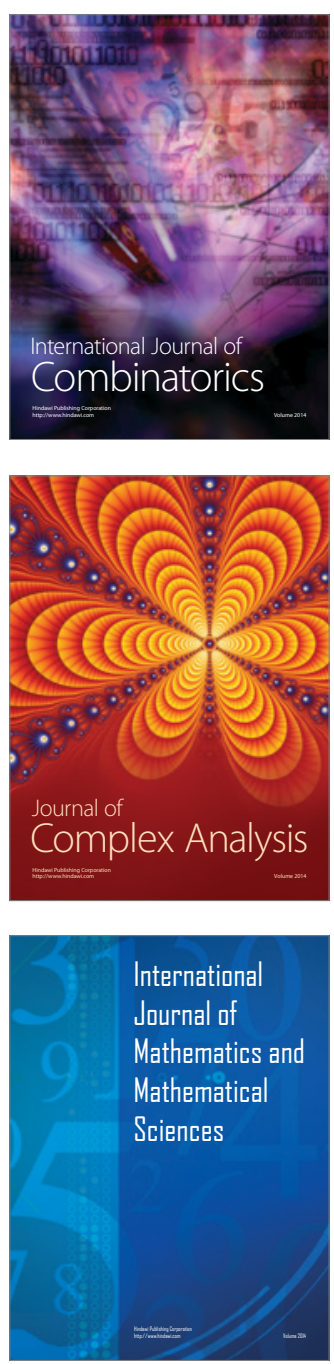
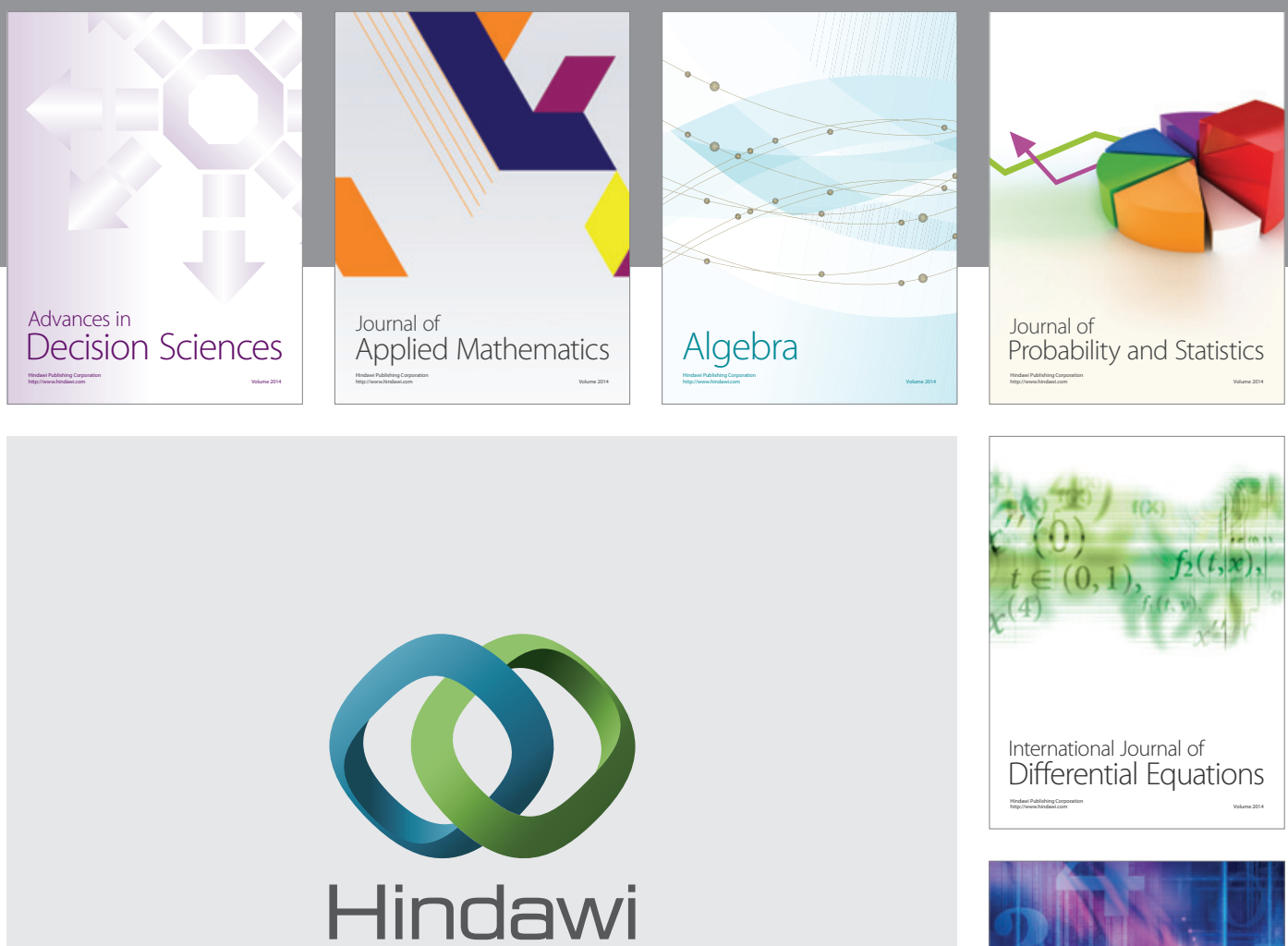

Submit your manuscripts at http://www.hindawi.com
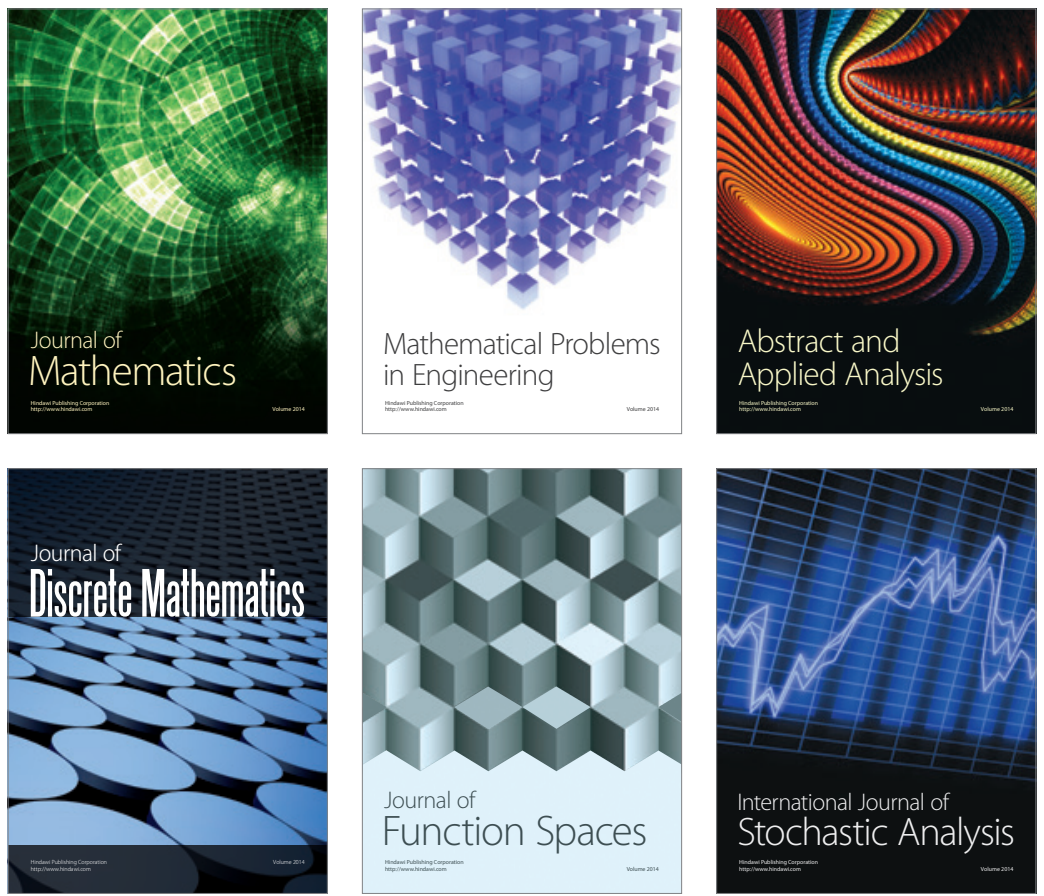

Journal of

Function Spaces

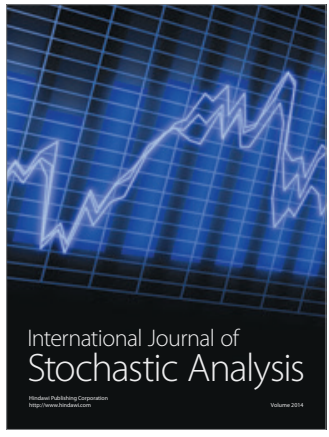

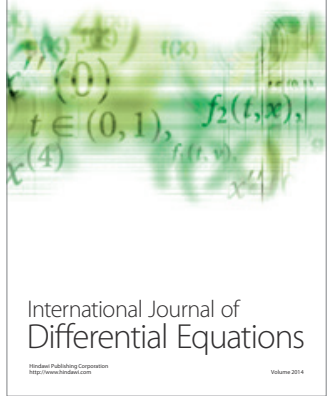
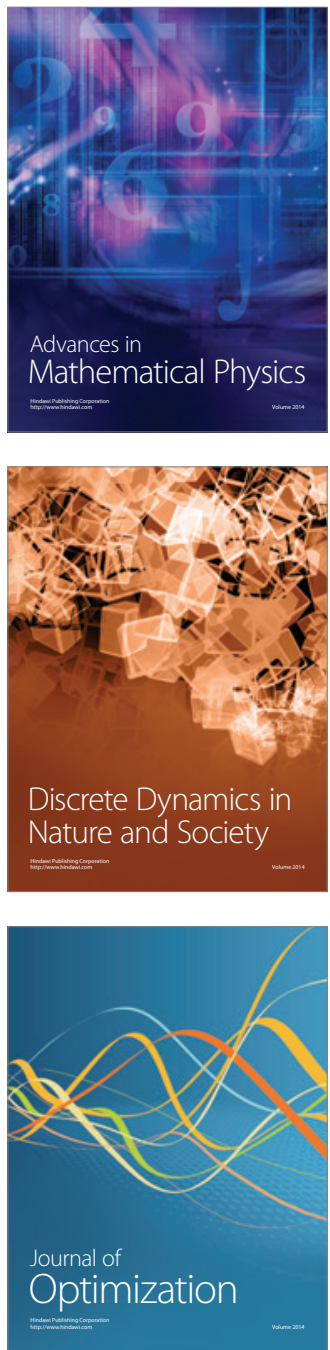